Rashid G. Alakbarov', Mammad A. Hashimov ${ }^{2}$

DOI: 10.25045/jpit.v07.i1.01

Institute of Information Technology of ANAS, Baku, Azerbaijan

${ }^{1}$ rashid@iit.ab.az, ${ }^{2}$ mhashimov@iit.ab.az

\title{
CLOUD COMPUTING TECHNOLOGIES: SERVICES, PROBLEMS AND APPLICATION AREAS
}

The paper analyzes the state of the art of cloud computing technologies, models and services. Several classes of computationally hard problems, which are solved by cloud technologies, are determined. Effective usage of computational and storage resources are studied.

Keywords: cloud computing, computational and storage resources, distributed systems, e-government, cloud services.

\section{Introduction}

Cloud Computing is widely used in the development of high performance distributed computing systems based on computer networks. Such high performance systems are established based on computer networks, which have high-speed communication channel. The use of highspeed communication channels ensures various organizations and enterprises to use cloud computing services more beneficially, in economic terms.

The surveys show that only $25-30 \%$ capacity of personal computer and 70-80\% resources of data center is used. In this case, the remaining unused computing and memory resources of PCs and data centers can be used to solve complex problems.

\section{Developing cloud-based distributed computing systems in network environment}

Cloud computing ensures the establishment and use of infrastructure and software of computer technology directly in the network environment. The user's data is stored and processed, in the cloud systems with the help of this technology, and at the same time, the browsers provide the launch of the processing programs and review results. Data processing and storage is realized by using clustering and virtualization of cloud technology infrastructure, computing and memory resources of computers.

This technology covers different types of computing concepts, which include numerous computers connected to the communication networks. It is similar to distributed computing, which enables the program on multiple computers connected over the network at the same time. Cloud technology is mainly based on the distribution of resources for solving large-capacity computations.

Cloud computing is quite broad term. The notion has as broad meaning as desktop computers, and covers from Windows management system to software.

Cloud technology provides the transfer of memory system and software resources of server computers of the enterprises into the clouds, i.e., it groups them. In general, this technology provides the user with computing and memory resources at the expense of internal resources on request.

Researches show that if enterprises use cloud technologies, they will not need to purchase and install computers, storage systems and software required for building data processing centers (DPC) at large expenditures. Experts think that, energy expenses, and the money, spent on technical equipment of large companies that use this technology, would be reduced by five times.

Many cloud experts predict that, in next five years, information technology infrastructure will be reformed. With the help of this technology, users will widely use cloud services (computing resources, software, data etc.) via the Internet through different computer equipment (PCs, laptops, smartphones, etc.). Gartner Group analysts call cloud technologies the most promising strategic technology, forecasting that within 5-7 years, most information technology will integrate into cloud technologies. According to their assessments, the share of cloud technology market will reach $\$ 200$ billion by 2016 [1].

In 2014, IDS (International Data Corporation, Framingham, Massachusetts, USA) forecasted that, the cost of cloud technologies would increase from 56.6 billion USD (2014) to 127.5 billion USD (2018) (Figure 1). In 2017, 17\% of annual expenditures spent on IT will fall on cloud [2]. 


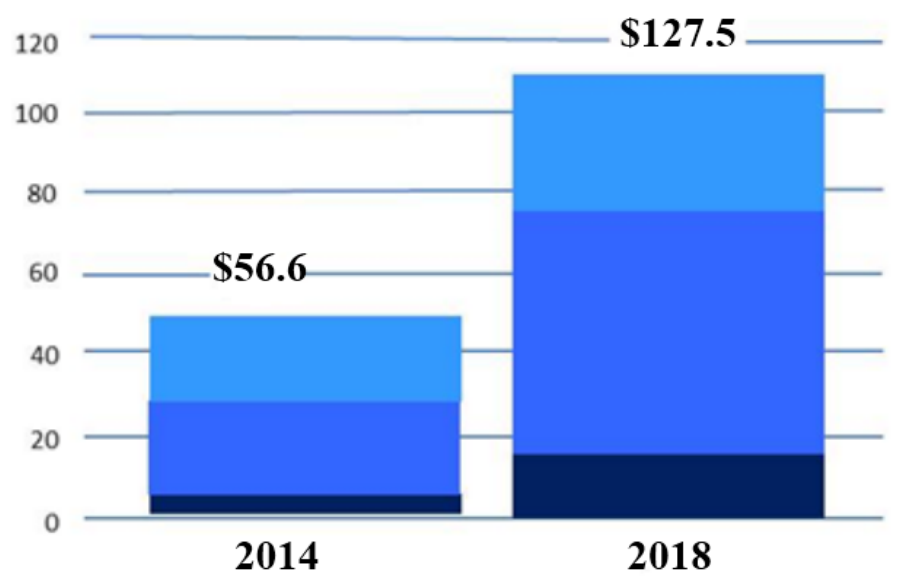

Platform-as-a-service Software-as-a-service Infrastructure-as-a-service

Figure 1. Dynamics of expenditures on cloud technologies

Competition in the field of cloud technologies has led to the emergence of free services. The rival companies Microsoft and Google are operating in this way. Both companies has introduced a number of services for the work with documents. Google offered Google Docs, and Microsoft Office Web Apps service [3].

In this case, both services are closely linked to mailing (Gmail and Hotmail respectively) and file storage. Therefore, the user is transferred from offline environment to online. Both Google and Microsoft support its online services in desktop computers, as well as mobile programming environment (note that, Google has developed Android operating system, Microsoft - Windows Phone 7). Apple, the main rival of both companies, promotes a similar concept (with a slightly different emphasis). That is MobileMe service. The service includes a tool for e-mail, calendar, address book, file storage, photo gallery and finding lost iPhone. In addition, Apple introduces the interaction between the Internet service and software package in (Mac running OS X) computer, phone, iPod, and iPad so that, there is no need to use a browser. When working on Mac, iPhone and iPad applications, all the information is stored in the clouds [3].

Three factors of Cloud technologies that attract users:

$\checkmark$ infinite possibilities of computational resources, that is, a user is free of pre-ordering and forecasting the necessary resources;

$\checkmark$ the absence of large expenditures in the early stages of the projects;

$\checkmark$ pay-as-you-go.

Advantages of Cloud technology:

$\checkmark$ Reduced need of PCs connected to the Internet for computing and memory resources;

$\checkmark$ Reduced costs of hardware and software, including CD and DVD, as all the data is stored in clouds. The users may use more comfortable and compact netbooks;

$\checkmark$ Availability of data anywhere and anytime, as the files are stored in clouds, even if you have forgotten some file. They are always in the clouds;

$\checkmark$ The data stored in clouds is automatically backed up across multiple servers, which are sometimes located on different continents. If the personal computer is stolen, the user does not lose valuable information, he/she is always able to get this information on any other computer.

$\checkmark$ Data Centers are managed by professionals, who support continuous operation of virtual machines. Even if the machine fails due to the distribution of many copies of the application, it will run, which proves high level of reliability of the system.

$\checkmark$ Providing the users with unlimited computing and memory resources (memory, processor, disk), and cloud may be flexible and scalable, that is, the resources are allocated and exported on request. 
Disadvantages of Cloud technology:

Stored data of a user depends on Cloud Computing service providers;

$\checkmark$ Reliability and security issues of communication channels;

$\checkmark$ Methods and standards that ensure quality service in this field have not been developed;

$\checkmark$ A user's computer must be connected to the Internet permanently;

$\checkmark$ High speed communication channel should be provided;

$\checkmark$ Implementation of some programs may take longer than their execution in local computers, and so on.

Models of cloud technologies and services

According to the definition of the cloud system, divided into $4[4,5]$ :

- $\quad$ Public clouds;

- $\quad$ Private clouds;

- $\quad$ Collective clouds;

- Hybrid clouds.

Public cloud - Subscriber of this cloud can be either company, or user. It offers solution of problems, which are impossible in other computing systems, at reasonable cost, and the development of web-sites or business-systems with measurement features. For example, Amazon EC2 and Simple Storage Service (S3), Google Apps / Docs, Salesforce.com, online services in Microsoft Office Web.

Private cloud - used and managed for only one organization. Everyone can use data, services and applications within the organization, but not outside of it.

Collective cloud - used and managed for only those organizations, which share common interests. Its structure can be distributed among one or more organizations, however, the essence is that required work is the same; the users require the same mission, strategy, security and speed.

Hybrid cloud - a model resulting from the combination and coordination of different cloud models (e.g., public, private and collective clouds). It is used by a number of internal and external cloud providers.

Cloud technology offers up to 10 services [6]:

Storage-as-a-service (SaaS) - Provides disk space on request. This resource may be located remotely and offers users the memory resources to store data.

Database-as-a-service (Daas) - Provides remote access data bank and database. The database appears as a base in local networks to the user.

Information-as-a-service (IaaS) - Provides remote access to data via the interface. This can be market data, credit information, verification and authentication of addresses.

Security-as-a-service (SAS) - Provides security services through the Internet. Despite the establishment of local security structure, some services as identification and certification, generation, storage and transmission of access keys are performed remotely.

Management / Governance-as-a-service (salary) - Provides remote management of other cloud services as, virtualization, managing access, implementation of specific policies (e.g., security). MaaS organizes business services.

Testing-as-a-service (TAAS) - Includes web servers, different types of services to provide access to local or remote testing.

Currently, the most commonly used cloud services are as follows:

IaaS (Infrastructure-as-a-service). Provides infrastructure development process. IaaS allows you to lease the infrastructure (computing and storage system). The resources include virtual servers providing computing and memory resources, as well as channels ensuring high-speed access to data storage system. In short, this level sets up computer infrastructure to resolve issues. Available IaaS services are Amazon S3 (Simple Storage Service), Amazon Elastic Computer Cloud (EC2), IBM's Blue Cloud and others. [6]. The user downloads Web browser in own computer and claims the clouds to solve the problem.

IaaS includes three main components [3]: 
1. Technical maintenance (servers, data storage systems, client systems, network equipment);

2. Operating systems and system software (virtualization and automation equipment, basic resource management);

3. Contact software (e.g., system management).

IaaS is based on virtualization technology, which allows the user to distribute the resources in accordance with the user's request, thereby increasing the efficiency of use of available computing capacity. In this case, the user consumes only necessary amount of disk space and other resources. In addition, IaaS offers the users all control functions in a single platform. Users do not have to manage the structure, but to operate the system and control the program.

$\mathrm{PaaS}$ (Platform-as-a-service). PaaS is a virtual platform ensuring the use of operational systems and special applications (Apache, My SQL and etc.), which are located on the virtual servers (consisting physical servers). For example, IBM IT Factory, Google App Engine, Force.com services.

This type of service provides the user with opportunity to place the functional programs purchased by the cloud infrastructure. Where, the user does not handle and examine the cloud structure such as network, or server. He/she just controls the installed functional application. For the development, testing, deployment and support of web-application PaaS provides all the operations as a single platform in a single integrated environment, thereby eliminates the expenditures spent on supporting different environments in separate stages [7].

SaaS - (Software as a service). The users are provided with software here. For example, Microsoft "Software Services" (email, video conferencing), Google Apps, Google Docs, and so on. The users are able to apply to the cloud technologies without any installations. The software applications run in the provider's server, which offers SaaS service, and send the result of calculations to the user. Therefore, the user does not purchase the software, but uses it only when he needs to solve the problem, and pays proper amount of money for the use.

Under the concept of SaaS, the user does not purchase the product, but he/she rents it. In addition, he/she uses just the functions he/she needs. For example, an application may be needed only once a year. And you're not going to use this program more than that. Thus, there is no need for unnecessary applications to capture any space on your computer. SaaS is based on the logical concept WaaS (WAAS - workspace as a service). In other words, the client is provided with fully equipped to virtual workspace to work with software [8].

\section{Complex problems to be solved via cloud technologies}

Currently, cloud technologies are considered to be the most promising technology to make more efficient use of DPC's resources.

Analysis of the characteristics of the solutions at data processing centers shows that, they can be attributed to either theoretical or applied sciences.

Complex problems to be solved in theoretical fields include:

- Analysis of prospective e-signature algorithms;

- Evaluation of symmetric encryption algorithms;

- Modeling oil and gas beds development processes;

- Application of mathematical models in the field of seismology;

- Extraction of new chemical substances and medicines;

- Extremely complex problem solution (economic, space research, chemical, physical, etc.);

- Information infrastructure building for the experiments in high-energy physics;

- Text mining in corporate information spaces and etc.

Complex problems to be solved in applied fields include:

- GIS-based real estate cadastre system creation (orthophoto, digital cadastre, topographic maps);

- State registry issues of the population;

- Registry of citizens' acts;

- e-data bank of notary documents; 
- Establishment of the Fundamental Scientific E-library;

- Establishment of e-database in the field of humanities;

- Information retrieval systems and so on.

The researches show that the complex issues solved in DPC are grouped in two classes according to their types, which require large computational resources and memory resources.

Complex issues that require large computing resources:

- Capturing, processing and archiving aerospace images of geographic areas;

- "e-Science" project realization based on international practices as part of "e-Government";

- Developing GIS-based hydro-meteorological and physical-geographical models of seas and oceans;

- Developing and researching mathematical models of climate processes in the atmosphere;

- Text mining in corporate information spaces;

- Diagnosis of diseases;

Complex issues that require large memory resources:

- e-Archive creation;

- Developing biometric identification system based on biometric technologies;

- Developing Monitoring Bank for Ecology and Natural Resources;

- Population Census;

- Information archiving;

- e-Atlas creation.

The efficient use resources in solving above-mentioned complex problems of the data processing centers mainly requires ensuring its optimal distribution. Currently, efficient use of the computing and memory resources in DPCs with the help of cloud technologies is intensively being investigated around the world. Such systems, which have large computing and memory resources, are established basing on computer networks with high-speed communication channels. Cloud technology ensures the organizations to use computing and memory resources of processing centers more efficiently.

Let's review some problems arising from the distribution of computing and memory resources among the users in the DPCs basing on cloud technologies.

Optimal distribution of the memory in the data processing center - Cloud Computing enables to scale and use physical resources (e.g. processor, storage and disk space) through the internet. In this case, the data processing and storage processes are considered as a type of service. The mentioned model provides that the storage resources allocated for any purpose is fully used and hold the space in the system as much as it is used. Accordingly, the user does not pay for reserved resource, but only for the actual resource use. And the provider reduces unnecessary purchase and installation of additional equipment; furthermore it can be able to offer the same service for lower price, which leads to greater user involvement in this type of services.

Distribution of the computing resources to the users in the data processing center Obviously, taking advantage of computing resources at the Data Centers does not only save the time, but also provides high quality problem solution. In this case, both the users, and the Data Center will get economic benefit. The benefit of users is the quality and efficient execution of the work. Actually, this efficiency is not expressed in accurate figures. However, experience shows that in many cases, the use of computational resources of data centers has no any alternatives to solve complex problems. Since the beginning of the use of data centers the optimization and efficiency became very vital. The perfect distribution of computing resources based on optimization and efficiency at data centers solves resource distribution problems of new users.

In recent years, the use of cloud technologies in many areas (e-government, e-education, elibrary, e-science, etc.) ensures more efficient use of network resources.

Application of Cloud technologies in e-Government system. Recently, multiple companies and users take advantage of a wide range of e-government services. This requires sustainable development of e-government basing on modern technologies. On the other hand, the cost of 
computer and network equipment used by the organizations to expand their network infrastructure, and the expenses spent in order to keep the efficiency of this structure are rising regularly. In this regard, the giant companies carry out extensive research to cut these costs by using existing resources, and search for new solutions to meet the requirements. The analysis of conducted studies shows that for the maximum use of the network resources cloud technology should be used. This technology ensures efficient use of network resources and Internet services.

The rapid growth of e-services of e-government system caused the expansion and complexity of the information exchange between government agencies. The presence of data processing centers in the state agencies and organizations, building them on various platforms requires groups of engineers and programmers to maintain multiple operating systems in operation, which is financially very expensive. At the same time, the use of different platforms and operating systems complicates their integration. To eliminate above-mentioned problems and to organize better quality service for users, cloud technologies are offered.

Cloud technology application in electronic library system. The use of Cloud technology in the development of digital libraries can be considered as a new stage, consecutively, it provides the creation of larger database, distribution of information over the network, ensuring the users new types of services, and reducing the expenditures. First, it is important to analyze the main models and services of this technology, and its benefits in order to understand the application of cloud technology in digital libraries.

If we look through technological capabilities of the middle of the previous century, we see that floppy discs were widely used in digital libraries as a space for data storage. However, it did not provide archiving large amounts of data. If we focus on recent years, we see that a lot of documents and data is digitalized and stored at "clouds" [4]. Currently, cloud technology is widely used in formation of digital library foundations, and in organization of services. One of the main objectives is acquisition of data and meta-data in the cloud-based holder, and automated management system must be developed in cloud environment.

\section{Conclusion}

The article analyzes the status of cloud technologies, its models, benefits and services, and solution of some problems addressed with the help of these technologies using data center resources. To take maximum advantage of computing and memory resources of data centers, optimal resource distribution should be provided through the application of cloud technology. Introducing cloud technologies to e-government and e-library system may improve the quality of service, reduce costs, achieve integration of services, and to organize centralized processing, archiving, and preservation of resources received from libraries, archives, museums and so on.

\section{References}

1. http://www2.itif.org/2013-cloud-computing-costs.pdf

2. http://www.idc.com/getdoc.jsp? containerId=prUS25219014

3. Introduction to Cloud Computing, http://www.intuit.ru/studies/courses/673/529/ lecture/11913.

4. Alguliyev R.M., Alekperov R.K. Cloud Computing: Modern State, Problems and Prospects // Telecommunications and Radio Engineering, 2013, vol.72, no.3, pp. 255-266.

5. Mell P., Grance T. The NIST definition of cloud computing, 2010, http://www.nist.gov/itl/ cloud/upload/cloud-def-v15.pdf

6. Alakbarov R.G., Hashimov M.A. About the prospects of cloud computing technologies in AzScienceNet // Problems of Information Technologies, 2012, No 2, pp. 30-36.

7. Alakbarov R.G., Hashimov M.A., Mustafayev T.I. Security issues of Cloud computing services and their solution ways // Problems of Information Technologies, 2014, No 2, pp. 33-39.

8. Soleimanian F., Hashemi S. Security Challenges in Cloud computing with More Emphasis on Trust and Privacy // International Journal of Scientific \& Technology Research, 2012, vol.1, no.6, pp.49-54. 